\title{
The correlation between blood lipids and clinicopathological features of breast cancer in young females
}

\author{
Wei Jin ${ }^{1 \#}$, Benjie Shan ${ }^{2 \#}$, Hu Liu ${ }^{1}$, Wenjuan $\mathrm{Li}^{1}$, Qianyu Zhang ${ }^{1}$, Shoubing Zhou ${ }^{1}$, Dandan $\mathrm{Hu}^{1}$, \\ Yueyin Pan $^{1}$
}

${ }^{1}$ The Fifth Ward of the Department of Tumor Chemotherapy, the First Affiliated Hospital of USTC, Division of Life Sciences and Medicine, University of Science and Technology of China \& Anhui Provincial Cancer Hospital, Hefei, China; ${ }^{2}$ Department of Tumor Chemotherapy, the First Affiliated Hospital of the USTC, Division of Life Sciences and Medicine, University of Science and Technology of China, Hefei, China

Contributions: (I) Conception and design: W Jin, B Shan; (II) Administrative support: Y Pan; (III) Provision of study materials or patients: W Jin; (IV) Collection and assembly of data: All authors; (V) Data analysis and interpretation: All authors; (VI) Manuscript writing: All authors; (VII) Final approval of manuscript: All authors.

\#These authors contributed equally to this work.

Correspondence to: Yueyin Pan. The Fifth Ward of the Department of Tumor Chemotherapy, the First Affiliated Hospital of USTC, Division of Life Sciences and Medicine, University of Science and Technology of China \& Anhui Provincial Cancer Hospital, Hefei 230031, China. Email: panyueyin@ustc.edu.cn; Hu Liu. The Fifth Ward of the Department of Tumor Chemotherapy, the First Affiliated Hospital of USTC, Division of Life Sciences and Medicine, University of Science and Technology of China \& Anhui Provincial Cancer Hospital, Hefei 230031, China. Email: drliuhu@yahoo.com.

Background: Breast cancer is becoming more common in young adults. The relationships between blood lipids and breast cancer have been widely studied in recent years. In our current study, we investigated the potential correlations between blood lipids and clinicopathological features of breast cancer in young females.

Methods: Fifty-nine young adults (40 years or younger) with pathologically confirmed invasive breast cancer that were treated in our center from October 2015 to March 2020 were enrolled in this study. These patients were divided into the negative group $(n=40$, with normal blood lipids) and positive group ( $n=19$, with abnormal blood lipids) according to the preoperative blood lipid profiles, and differences in the clinicopathological features were compared between these two groups.

Results: Compared with the negative group, the positive group had a significantly higher rate of lymph node positivity $(\mathrm{P}=0.034)$; compared with the positive group, the negative group had a significantly higher rate of HER2 positivity $(\mathrm{P}=0.029)$. However, these two groups showed no significant difference in tumor size, molecular type, clinical stage, histological grade, tumor thrombus, and Ki-67 index (P values were 0.071, $0.227,0.593,0.396,0.198$, and 0.593 , respectively).

Conclusions: Blood lipid level has a certain correlation with lymph node metastasis and HER2 expression in young breast cancer patients. Therefore, blood lipid levels has a certain reference value in the clinical treatment of breast cancer.

Keywords: Breast cancer; young females; blood lipids; clinicopathological features.

Submitted Jun 03, 2020. Accepted for publication Sep 18, 2020.

doi: $10.21037 / g s-20-616$

View this article at: http://dx.doi.org/10.21037/gs-20-616 


\section{Introduction}

Breast cancer is one of the most common and fatal female malignant tumors, and epidemiological data suggest it affects middle-aged and older women $(1,2)$. In recent years, however, more breast cancer cases have been found in young females $(3,4)$. As the deadliest malignancy in young women, breast cancer has a remarkably poorer prognosis in young females than in older women (5). Although many studies have investigated the pathogenesis and epidemiological characteristics of breast cancer in young females, there is no widely recognized definition of breast cancer in this population. In most published articles, young women with breast cancer were those under 40 years of age (6-8). Therefore, this study defines the upper age limit for young adults with breast cancer as 40 years. Lipid abnormality has become prevalent in modern society. Research has shown abnormal blood lipid levels are correlated with the prognosis of breast cancer. The prognosis of breast cancer patients is closely related to its clinicopathological features, especially young breast cancer patients. However, the relationship between blood lipids and clinicopathological characteristics of young breast cancer has not been studied yet. Here, we collected the blood lipid profiles and clinicopathological data from 59 female breast cancer patients aged 40 years and younger and analyzed the potential differences in the clinicopathological features between patients with or without lipid abnormality. We present the following article in accordance with the MDAR reporting checklist (available at http://dx.doi.org/10.21037/gs-20-616).

\section{Methods}

\section{Clinical data}

Fifty-nine patients who underwent surgical treatment in our center from October 2015 to March 2020 were enrolled in our study. These patients had pathologically confirmed invasive breast cancer and were aged 40 years or younger. All patients met the following inclusion criteria: (I) the diagnosis was pathologically confirmed after the surgery; (II) the tumor was treatment-naive before surgery, and blood lipid profiles were retrieved; and (III) the postoperative clinicopathological data including pathological type, tumor size, lymph node metastasis, estrogen receptors (ER), progesterone receptors (PR), human epidermal growth factor receptor 2 (HER2), and cell proliferation marker Ki67 were complete. All procedures performed in this study involving human participants were in accordance with the
Declaration of Helsinki (as revised in 2013). The ethics committee approved the study of our hospital (No. 2020ZNW-01) and due to the retrospective nature of the study, informed consent was waived.

\section{Pathological stages}

The breast was staged according to the American Joint Committee on Cancer's TNM classification (8th edition).

\section{Blood lipids}

Measurements of blood lipids were obtained from the Clinical Laboratory of our center. The "normal" or "abnormal" results were based on the upper and lower thresholds of the blood lipids. According to the Guidelines on Adult Lipid Control in China (2016 Edition), we defined dyslipidemia based on the following criteria: the level of total cholesterol, triglyceride, low-density lipoprotein, and/ or Apo-B is higher than the normal range or the level of high-density lipoprotein and/or Apo-A1 is lower than the normal range. Patients were divided into a positive group (with abnormal blood lipids) and a negative group (with normal blood lipids) according to the blood lipid levels.

\section{Pathological data}

The pathological classification criteria were based on the WHO Classification of Tumors of the Female Reproductive Organs (4th Edition). The expressions of ER, PR, and HER2 were defined according to ASCO/CAP laboratory testing guidelines. For ER and PR, nuclear staining in $\geq 1 \%$ of the tumor cells was positive. HER2 status was assessed using the following criteria: - (no membrane staining), 1+ (weak or incomplete staining of any proportion of tumor cells), $2+$ (weak but complete membrane staining in $\geq 10 \%$ of tumor cells or incomplete membrane staining, or strong, complete membrane staining in $<30 \%$ of tumor cells), and $3+$ (strong, complete membrane staining in $\geq 30 \%$ of tumor cells). All patients with an HER2 score of $2+$ were subjected to fluorescent in situ hybridization (FISH). HER2 was defined as positive if the immunohistochemical score was $3+$ or if the immunohistochemical score was $2+$, but FISH showed HER2 gene amplification; HER2 was defined as negative if the immunohistochemical score was - or $1+$ or if the immunohistochemical score was 2+, but FISH showed no HER2 gene amplification. The proliferation marker $\mathrm{Ki}-67$ was defined as the percentage of tumor cells 
positive for Ki-67 nuclear staining. The breast cancer was divided into four molecular subtypes based on the immunohistochemical measures of ER, PR, HER2, and Ki-67: Luminal A: ER and/or PR positive, HER2 negative, and Ki-67 <14\%; Luminal B: ER and/or PR positive, HER2 negative, and $\mathrm{Ki}-67 \geq 14 \%$; or, ER and/or PR positive and HER2 positive; HER2 over-expression: ER and PR negative and HER2 positive; and triple-negative: ER, PR, and HER-2 were negative.

\section{Treatment and follow-ups}

All patients received surgical therapy in our center. The surgical procedures included breast-conserving surgery, modified radical mastectomy, and skin-sparing mastectomy + breast reconstruction. After the surgery, chemotherapy, radiotherapy, targeted therapy, and/or endocrine therapy were offered according to the pathological findings and clinical stage. Some patients received these treatments recently, and therefore their disease-free survival (DFS) and overall survival (OS) were unfollowed up.

\section{Statistical analyses}

Data were processed using SPSS 17.0 software. Qualitative data are presented using frequencies and contingency tables. Data in two groups were compared using a chi-square test. A P value of $<0.05$ was considered significantly different.

\section{Results}

\section{Age compositions}

These 59 patients aged $23-40$ years [mean: $(35.93 \pm 4.19)$ years], and the specific age composition was: $\leq 25$ years, 2 patients (3.4\%); $26-30$ years, 4 patients $(6.8 \%) ; 31-35$ years, 16 patients (27.1\%); and $36-40$ years, 37 patients $(62.7 \%)$.

\section{Clinicopathological features}

Among these 59 breast cancer patients, 14 patients (23.7\%) were in stage I, $34(57.6 \%)$ in stage II, and $11(18.6 \%)$ in stage III. There were 35 patients (59.3\%) with lymph node metastasis and $24(40.7 \%)$ without metastasis. Forty-four patients $(74.6 \%)$ were luminal A/B, $9(15.3 \%)$ were triplenegative, and $6(10.2 \%)$ were overexpression. Histologically, 39 patients $(66.1 \%)$ were in grade I-II and $20(33.9 \%)$ in grade III. HER2 expression was positive in 21 patients
(35.6\%) and negative in 38 patients $(64.4 \%)$. Tumor thrombus was positive in 24 cases (40.7\%) and negative in 35 cases $(59.3 \%)$. The Ki-67 proliferation index was $\leq 30 \%$ in 25 cases $(42.4 \%)$ and $>30 \%$ in 34 cases $(57.6 \%)$.

\section{Relationship between blood lipid levels and clinicopathological features in breast cancer patients}

Of these 59 patients, blood lipid levels were normal in 40 patients and abnormal in 19 cases. Chi-square test was performed to compare the differences in tumor size, lymph node metastasis, molecular type, clinical stage, histological grade, HER2 positive expression, tumor thrombus, and $\mathrm{Ki}-67$ expression. Compared with group A, group B had a significantly higher rate of positive lymph nodes $(\mathrm{P}=0.034)$; compared with group B, group A had a significantly higher rate of HER2 positivity $(\mathrm{P}=0.029)$. However, these two groups showed no significant difference in tumor size, molecular type, clinical stage, histological grade, tumor thrombus, and Ki-67 index (P values were 0.071, 0.227, $0.593,0.396,0.198$, and 0.593 , respectively) (Table 1).

\section{Discussion}

In recent years, multidisciplinary treatment strategies, including surgery, chemotherapy, radiotherapy, targeted therapy, and endocrine therapy have been applied in the management of breast cancer, which has dramatically improved the outcomes of breast cancer patients, with some patients achieving prolonged survival time (9). However, the number of new breast cancer cases is still rising annually, especially in young females (10). Breast cancer is more invasive and more likely to recur in young patients. Disease relapse is associated with lower treatment response, faster disease progression, and shorter survival, resulting in an extremely poor prognosis for these patients. Therefore, further investigations on the clinicopathological characteristics of young patients with breast cancer are urgently needed. Research has shown the subtypes and grades of breast cancer in young women are different from those in elderly populations. Young patients suffer from a more aggressive phenotype. In particular, HER2-positive breast cancer is more common for young women (11). Our current study retrospectively analyzed the clinicopathological features of 59 young patients with breast cancer, among whom postoperative pathology confirmed positive HER2 expression in $35.6 \%(n=21)$ of the patients, which was higher than the average percentages in the 
Table 1 Relationship between blood lipids and clinicopathological features

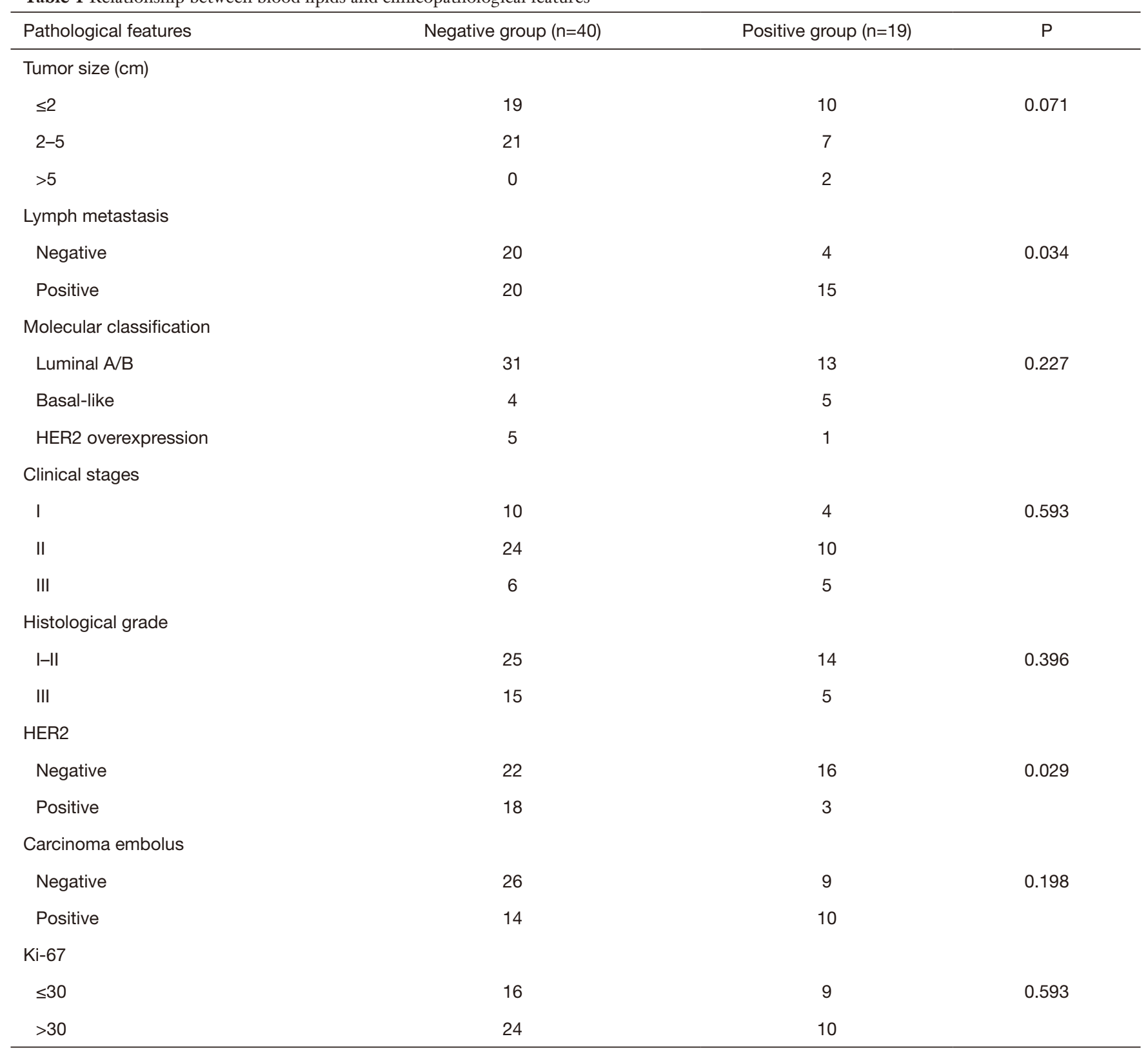

HER2, human epidermal growth factor receptor 2.

general breast cancer patients and was consistent with the findings in other literature $(12,13)$.

Abnormalities of lipid metabolism are common in populations and are the known risk factors for cardiovascular diseases (14). The relationships between abnormal blood lipid metabolism and malignant tumors (especially breast cancer) have been widely studied worldwide $(15,16)$. Blood lipids and lipoproteins are affected by environmental factors, including body weight and diet. Bodyweight and diet are closely related to the risk factors of breast cancer, suggesting that lipids may be related to breast cancer by affecting breast cancer risk factors. Some recent studies have explored the relationships between lipids and breast cancer. For example, a large prospective study in the Republic of Korea showed high cholesterol increased the risk of breast cancer (17). Another study showed the average levels of 
total cholesterol, low-density lipoprotein, and high-density lipoprotein were lower in patients with malignant tumors than in patients with non-malignant tumors (18). A clinical study enrolling 38,823 Norwegian women, the first survey showed there was a positive correlation between HDL-C and tumor occurrence in breast cancer patients before menopause. However, in the second survey, HDL-C was uncorrelated with the occurrence of breast cancer; rather, low HDL-C is associated with increased postmenopausal breast cancer risk (19). In one of our previous cell experiments, we found the lipid-lowering drug simvastatin could suppress the proliferation of breast cancer MCF7 cells, and further research suggested that simvastatin could induce the cell cycle arrest and apoptosis of tumor cells by up-regulating the expression of Bax protein and down-regulating the expression of Bcl-2 protein. Thus, the relationships between blood lipids and breast cancer are complex and deserve further investigations.

Up to now, no study has reported the correlation between blood lipid levels and clinicopathological features of breast cancer in young populations. In our current study, tumor size, molecular type, clinical stage, histological grade, tumor thrombus, and Ki-67 index were not significantly different between patients with normal and abnormal blood lipids. Compared with patients with normal blood lipids, those with abnormal blood lipids had a significantly higher rate of positive lymph nodes. Thus, positive lymph nodes suggest the tumor is in a more advanced stage and is at higher risk of recurrence. Clinically, positive lymph nodes are one criterion for initiating postoperative adjuvant radiotherapy and chemotherapy, suggesting that breast cancer patients with dyslipidemia may have a worse prognosis.

The HER2 protein is a member of the epidermal growth factor receptor family having tyrosine kinase activity. The HER 2 gene is on chromosome $17 \mathrm{q} 12-21.32$. Patients with positive HER2 protein expression or gene amplification account for $15-20 \%$ of breast cancer cases (20). HER2, as the most critical gene of breast cancer, takes part in the growth, proliferation, invasion, and migration of breast cancer. As an indicator of targeted adjuvant therapy, HER2 gene amplification can predict the prognosis of patients $(21,22)$. The HER2 positivity rate was higher in our patients with normal blood lipid levels than in those with abnormal blood lipid levels, suggesting that patients with normal blood lipids have a higher chance of receiving anti-HER2 treatment. The relationship between HER2 and blood lipids is worth exploring. Cha et al. (23) showed that HER2 can induce transcriptional activation of leptin in MCF10A human mammary epithelial cell-line through p38 mitogenactivated protein kinase signaling. The results indicate that leptin is closely related to HER2. Another study found that HER2 was positively correlated with various obesityrelated parameters, such as triglycerides, hyperglycemia, and BMI (24). The abnormal expression of leptin can lead to disorders of blood lipid metabolism. Therefore, HER2 and blood lipids may interact through leptin.

To sum up, the blood lipid level has a certain correlation with lymph node metastasis and HER2 expression in young breast cancer patients. Therefore, the levels of blood lipids may be informative when offering individualized treatment for these patients. However, our current study was limited by its single-center retrospective design. Due to the small sample size, the study did not analyze total serum cholesterol (TC), triglyceride (TG), and high-density lipoprotein (HDL) cholesterol separately. The conclusion needs to be confirmed by a large sample study.

\section{Acknowledgments}

Funding: This paper was supported by Special funds for basic scientific research operating expenses of central universities (Fund No.WK9110000058),Natural Science Foundation of Anhui Province, general project (Fund No.1908085MH260),Hefei independent innovation policy "borrow and replace" project (Fund No. J2018Y01), The youth Scientific Research Fund project of the First Affiliated Hospital of USTC and Anhui Tumor Hospital in 2018 (Fund No. 2018YJQN011).

\section{Footnote}

Reporting Checklist: The authors have completed the MDAR reporting checklist. Available at http://dx.doi.org/10.21037/ gs-20-616

Data Sharing Statement: Available at http://dx.doi. org/10.21037/gs-20-616

Conflicts of Interest: All authors have completed the ICMJE uniform disclosure form (available at http://dx.doi. org/10.21037/gs-20-616). The authors have no conflicts of interest to declare.

Ethical Statement: The authors are accountable for all aspects of the work in ensuring that questions related to the accuracy or integrity of any part of the work are 
appropriately investigated and resolved. All procedures performed in this study involving human participants were in accordance with the Declaration of Helsinki (as revised in 2013). The ethics committee approved the study of our hospital (No. 2020-ZNW-01) and due to the retrospective nature of the study, informed consent was waived.

Open Access Statement: This is an Open Access article distributed in accordance with the Creative Commons Attribution-NonCommercial-NoDerivs 4.0 International License (CC BY-NC-ND 4.0), which permits the noncommercial replication and distribution of the article with the strict proviso that no changes or edits are made and the original work is properly cited (including links to both the formal publication through the relevant DOI and the license). See: https://creativecommons.org/licenses/by-nc-nd/4.0/.

\section{References}

1. DeSantis CE, Ma J, Gaudet MM, et al. Breast cancer statistics, 2019. CA Cancer J Clin 2019;69:438-51.

2. Kosaka Y, Nishimiya H, Kikuchi M, et al. Historical progress of the 8th edition of the American Joint Committee on Cancer (AJCC) Cancer Staging Manual in patients with breast cancer. Transl Cancer Res 2019;8:719-21.

3. Thomas A, Rhoads A, Suhl J, et al. Incidence and Survival by Human Epidermal Growth Factor Receptor 2 Status in Young Women With Stage I-III Breast Cancer: SEER, 2010-2016. Clin Breast Cancer 2020;20:e410-22.

4. Anastasiadi Z, Lianos GD, Ignatiadou E, et al. Breast cancer in young women: an overview. Updates Surg 2017;69:313-7.

5. Zimmer AS, Zhu K, Steeg PS, et al. Analysis of breast cancer in young women in the Department of Defense (DOD) database. Breast Cancer Res Treat 2018;168:501-11.

6. Spring L, Greenup R, Niemierko A, et al. Pathologic Complete Response After Neoadjuvant Chemotherapy and Long-Term Outcomes Among Young Women With Breast Cancer. J Natl Compr Canc Netw 2017;15:1216-23.

7. Eccles DM, Li N, Handwerker R, et al. Genetic testing in a cohort of young patients with HER2-amplified breast cancer. Ann Oncol 2016;27:467-73.

8. Ruggeri M, Pagan E, Bagnardi V, et al. Fertility concerns, preservation strategies and quality of life in young women with breast cancer: Baseline results from an ongoing prospective cohort study in selected European Centers. Breast 2019;47:85-92.

9. Sanchez AM, Franceschini G, Terribile D, et al.
Personalized medicine in breast cancer: a step forward. Ann Transl Med 2019;7:S138.

10. Shoemaker ML, White MC, Wu M, et al. Differences in breast cancer incidence among young women aged 20-49 years by stage and tumor characteristics, age, race, and ethnicity, 2004-2013. Breast Cancer Res Treat 2018;169:595-606.

11. Greally M, Kielty J, Watson GA, et al. Where youth matters-clinicopathologic characteristics and emerging trends in treatment and outcomes in young Irish women with breast cancer. Ir J Med Sci 2019;188:59-67.

12. Arnould L, Penault-Llorca F, Dohollou N, et al. [Breast cancer in young women. Histological and prognostic specificities: how are they different from older women?]. Bull Cancer 2019;106:S10-8.

13. Johansson ALV, Trewin CB, Hjerkind KV, et al. Breast cancer-specific survival by clinical subtype after 7 years follow-up of young and elderly women in a nationwide cohort. Int J Cancer 2019;144:1251-61.

14. Huth PJ, Park KM. Influence of dairy product and milk fat consumption on cardiovascular disease risk: a review of the evidence. Adv Nutr 2012;3:266-85.

15. Nowak C, Ärnlöv J. A Mendelian randomization study of the effects of blood lipids on breast cancer risk. Nat Commun 2018;9:3957.

16. His M, Dartois L, Fagherazzi G, et al. Associations between serum lipids and breast cancer incidence and survival in the E3N prospective cohort study. Cancer Causes Control 2017;28:77-88.

17. Kitahara CM, Berrington de González A, Freedman $\mathrm{ND}$, et al. Total cholesterol and cancer risk in a large prospective study in Korea. J Clin Oncol 2011;29:1592-8.

18. Li X, Liu ZL, Wu YT, et al. Status of lipid and lipoprotein in female breast cancer patients at initial diagnosis and during chemotherapy. Lipids Health Dis 2018;17:91.

19. Furberg AS, Veierød MB, Wilsgaard T, et al. Serum highdensity lipoprotein cholesterol, metabolic profile, and breast cancer risk. J Natl Cancer Inst 2004;96:1152-60.

20. Di Oto E, Brandes AA, Cucchi MC, et al. Prognostic impact of HER-2 Subclonal Amplification in breast cancer. Virchows Arch 2017;471:313-9.

21. Wu Z, Xu S, Zhou L, et al. Clinical significance of quantitative HER2 gene amplification as related to its predictive value in breast cancer patients in neoadjuvant setting. Onco Targets Ther 2018;11:801-8.

22. Buckley NE, Forde C, McArt DG, et al. Quantification of HER2 heterogeneity in breast cancer-implications for identification of sub-dominant clones for personalised 
treatment. Sci Rep 2016;6:23383.

23. Cha Y, Kang Y, Moon A. HER2 induces expression of leptin in human breast epithelial cells. BMB Rep 2012;45:719-23.

Cite this article as: Jin W, Shan B, Liu H, Li W, Zhang Q, Zhou S, Hu D, Pan Y. The correlation between blood lipids and clinicopathological features of breast cancer in young females. Gland Surg 2020;9(5):1443-1449. doi: 10.21037/gs-20-616
24. Memon AA, Bennet L, Zöller B, et al. Circulating human epidermal growth factor receptor 2 (HER2) is associated with hyperglycaemia and insulin resistance. J Diabetes 2015;7:369-77. 